\title{
Carbon Monoxide Poisoning during Hurricane Sandy in Affected New York State Counties
}

\author{
Jian-Hua Chen*, Ursula Lauper, Cristian Pantea, Shao Lin and Hwa-Gan Chang \\ Division of Epidemiology, New York State Department of Health, Albany, NY, USA
}

\section{Objective}

1) To identify carbon monoxide $(\mathrm{CO})$ poisoning in three most affected New York State (NYS) counties (Nassau, Suffolk, and Westchester) during and immediately after Hurricane Sandy with hospital emergency department (ED) chief complaint data reported daily to the New York State Department of Health (NYSDOH). 2) To explore the relationship between power outage and the numbers of CO-related ED visits (CO-EDs).

\section{Introduction}

$\mathrm{CO}$ poisoning is a leading cause of mortality and morbidity in disaster and post-disaster situations, when widespread power outages most likely occur $(1,2)$. The NYSDOH Syndromic Surveillance System receives daily ED visit chief complaint data from 140 NYS (excluding New York City) hospitals. Daily power outage data are available from the NYS Department of Public Service (NYSDPS). These data can be used to estimate the risk of CO-EDs and provide useful information for public health situational awareness and emergency response management during disaster events.

\section{Methods}

This was a retrospective study in which the daily CO-EDs were identified by searching CO-related keywords in chief complaints of the ED visits from the affected three counties during the Sandy period (October 29 - November 27, 2012). Data on daily maximum number of households without power during the hurricane in each of the three affected counties were obtained from NYSDPS. The correlation between CO-EDs and maximum number of households without power was estimated with a log-transformed linear regression model. A negative binomial model was used to examine the risk of CO-EDs by the maximum households affected by power outages each day during the month following Hurricane Sandy. Data were analyzed using SAS 9.3 and the p-value for testing statistical significance was set at 0.05 .

\section{Results}

A total of $188 \mathrm{CO}$-EDs were identified in the three affected counties during the study period. Overall, the number of CO-EDs increased during the hurricane period. Coinciding with the power outage in the areas, the CO-EDs increase peaked at 3-4 days after the hurricane made landfall in NYS on October 29 (Figure 1). Statistical analysis showed that there was a significant correlation between the maximum number of households without power and the number of CO- EDs such that $53 \%$ of the variance of CO-EDs can be explained by the power outage $(\mathrm{p}<0.0001)$. The risk ratio of $\mathrm{CO}$ poisoning was more than three times as great on days of significant power outage (i.e. when more than 200,000 households were without power), compared with days of insignificant power outage - defined as 10,000 or fewer households (Table 1). The higher the number of households without power, the higher the risk ratio of $\mathrm{CO}$ poisoning.

\section{Conclusions}

$\mathrm{CO}$ exposures can be identified with ED chief complaint data, and the risk of CO-EDs can be estimated using power outage data.
If ED data and/or power outage data are available in a timely base, they can provide valuable $\mathrm{CO}$ exposure information for situational awareness and emergency management during and after disasters. The study results also support that power outage is an underlying cause that indirectly leads to $\mathrm{CO}$ exposures. Education on proper use of alternative power and heating sources should be part of public programs designed to prevent future disaster-associated $\mathrm{CO}$ poisoning.

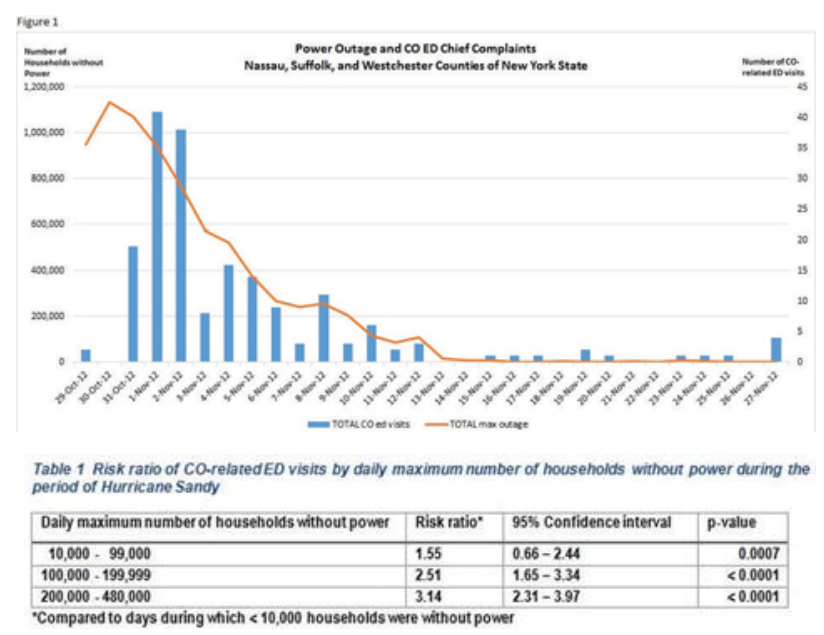

\section{Keywords}

CO poisoning; event syndromic surveillance; disaster situational awareness; power outage; chief complaint

\section{References}

1. Hampson NB, Stock AL,. Storm-related carbon monoxide poisonings: Lessons learned from recent epidemics. Undersea Hyperb Med 2006; 33:257-63.

2. Iqbak S, Clower JH, Hernandez SA, Damon SA, Yip FY. A review of disaster-related carbon monoxide poisoning: surveillance, epidemiology, and opportunities fro pervention. Am J Public Health 2012;102:1957-63.

\author{
*Jian-Hua Chen \\ E-mail: jianhua.chen@health.ny.gov
}

